Bio - grafia. Escritos sobre la Biología y su Enseñanza. ISSN 2027

Edición Extraordinaria. p.p. 1275- 1288

Memorias del VIII Encuentro Nacional de Experiencias en Enseñanza de la Biología y la Educación Ambiental. III Congreso Nacional de Investigación en Enseñanza de la Biología.

\title{
UNA DOCENCIA UNIVERSITARIA AMBIENTALIZADA Y MEDIADA POR LAS TIC
}

\section{A UNIVERSITY TEACHING AMBIENTALIZADA AND MEDIATED BY ICT}

\author{
CLAUDIA MARÍA CARDONA LONDOÑO ${ }^{1}$
}

cmcardona@udistrital.edu.co

Resumen

Pensar en la formación integral de los docentes universitarios es confirmar la urgente necesidad de transformar la praxis pedagógica del docente incorporando las mediaciones tecnológicas y la inclusión de la dimensión ambiental en correspondencia con las exigencias del tiempo actual y en sintonía con los nuevos desafíos del mundo cambiante que permita la transformación del proceso de enseñanza en el aula. La presente comunicación busca esquematizar la idea inicial del proyecto de tesis doctoral titulado "Una docencia Universitaria Ambientalizada y mediada por TIC", donde el principal problema es la búsqueda de las relaciones entre la inclusión de la dimensión ambiental y las TIC, cómo una nueva consideración en la política de formación docente.

De la metodología planteada se resalta la necesidad de trabajar sobre un diseño pedagógico para la presentación de contenidos en ambientes virtuales de aprendizaje, la estructura de contenidos y los roles del docente virtual ambientalizado. La formación permanente del docente universitario, será un compromiso de apropiación del conocimiento especializado con característica de una conciencia crítica, humanista, reflexiva, emancipadora que

\footnotetext{
${ }^{1}$ Estudiante del DIE-UD. Docente de Planta de la UD-FJC- FAMARENA-Ing. Forestal
} 
Bio - grafia. Escritos sobre la Biología y su Enseñanza. ISSN 2027

Edición Extraordinaria. p.p. 1275- 1288

Memorias del VIII Encuentro Nacional de Experiencias en Enseñanza de la Biología y la Educación Ambiental. III Congreso Nacional de Investigación en Enseñanza de la Biología.

construye realidades socioculturales, políticas y éticas en los diversos contextos de estudio y aplicación.

Se incluye una primera aproximación a un marco conceptual y contextual local, desde la política TIC para Colombia y desde el reconocimiento de una Universidad Sustentable, donde la tecnología será la mediación tecnológica y de elementos de la dimensión ambienta se verán como parte de la apropiación del mundo y su entorno de vida.

Palabras Clave: Formación del Docente, Mediaciones con TIC, Docencia Ambientalizada

Abstract

Think about the integral formation of university teachers is to confirm the urgent need to transform the pedagogical praxis teacher incorporating technological mediations and the inclusion of the environmental dimension in correspondence with the demands of the present time and in tune with the new challenges of the changing world that allows the transformation of the learning process in the classroom. This communication aims outlining your initial idea for the doctoral thesis project entitled "a university teaching Ambientalizada and mediated by ICT", where the main problem is the search for relations between the inclusion of the environmental dimension and ICT, such as a new account in the policy of teacher training. The methodology is highlighted the need to work on a pedagogical design for presentation of content in virtual learning environments, the content structure and the roles of the teacher virtual the environment. 
Bio - grafia. Escritos sobre la Biología y su Enseñanza. ISSN 2027

Edición Extraordinaria. p.p. 1275- 1288

Memorias del VIII Encuentro Nacional de Experiencias en Enseñanza de la Biología y la Educación Ambiental. III Congreso Nacional de Investigación en Enseñanza de la Biología.

The continuing education of university teachers, will be a commitment of appropriation of the specialized knowledge with characteristic of a critical awareness, humanist, reflexive, emancipatory that builds sociocultural, political and ethical in the various contexts of study and application.

It includes a first approximation to a conceptual framework and contextual local, from the ICT policy for Colombia and since the recognition of a University Sustainable, where the technology will be the mediation technology and elements of the dimension environmental will be seen as part of the appropriation of the world and its living environment.

Key Words: Teacher Training, mediations with ICT, Teaching the environment 
Bio - grafia. Escritos sobre la Biología y su Enseñanza. ISSN 2027

Edición Extraordinaria. p.p. 1275- 1288

Memorias del VIII Encuentro Nacional de Experiencias en Enseñanza de la Biología y la Educación Ambiental. III Congreso Nacional de Investigación en Enseñanza de la Biología.

\section{Introducción}

El e-learning es un espacio de aprendizaje que utiliza TIC. Se trata de una formación bajo el uso de redes el cual permite acceso a información y el aprendizaje mediante elementos tecnológicos generalmente usando internet, disponiendo de materiales como libros electrónicos, audio, video entre otros. Desde nuestro punto de vista deberíamos abrir una nueva etapa que asuma que los procesos de enseñanza-aprendizaje son sistémicos y que, en ese caso, todas las variables deben adaptarse a las características de los estudiantes, que en este caso son Docentes Universitarios, con un saber previo en una acción formativa integral que incluye el conocimiento y dominio de las TIC y la inclusión de la dimensión ambiental en su discurso pedagógico y didáctico. (Cabero J. , 2006).

En el caso del uso del e-learning en la educación superior permite identificar una serie de problemas sobre todo en el uso pedagógico o buena práctica de los ambientes virtuales de aprendizaje, donde los docentes deben desarrollar habilidades propias de su ejercicio profesional, con un nuevo rol y un contexto de estudio particular, la obligación de asumir un lugar en la historia de la sustentabilidad ambiental, como una responsabilidad social compartida, está en la idea de una formación permanente e integral, que involucre la realidad social consolidada en reflexiones del saber cotidiano y significativo, es desde allí donde se hace la verdadera construcción social de la realidad universitaria, en este sentido la teoría del conocimiento de Schutz, 1994 "Stock de conocimiento" (Schutz, 1994), establece que cada individuo se integra a partir de las tipificaciones del mundo y del sentido común, estableciendo una categorías o características de realidades múltiples que 
Bio - grafia. Escritos sobre la Biología y su Enseñanza. ISSN 2027

Edición Extraordinaria. p.p. 1275- 1288

Memorias del VIII Encuentro Nacional de Experiencias en Enseñanza de la Biología y la Educación Ambiental. III Congreso Nacional de Investigación en Enseñanza de la Biología.

permite trabajar en la autoreflexión entorno principalmente en su quehacer docente y pedagógico, los aspectos tales como en nuestro caso las TIC y la inclusión de la dimensión ambiental.

Es responsabilidad de las Universidades del país la implementación y desarrollo de un Plan Estratégico en TIC que enfatiza en todos los escenarios desde donde se debe abordar el uso y apropiación tecnológica. No basta con la adquisición de nuevas tecnologías, redes y sistemas de computación, sin que exista previamente un proceso de formación y desarrollo de competencias tecnologías y la generación de una cultura informática propia de la comunidad académica, el valor agregado está en la inclusión de la dimensión ambiental.

La educación superior tiene cierta responsabilidad en el marco del desarrollo sostenible y están moralmente obligadas a enseñar y producir conocimientos, preparando tecnólogos y profesionales que favorezcan modelos de sustentabilidad ambiental, para solucionar los problemas de la sociedad haciéndose cogestores no solo de sus estudiantes sino también de las comunidades y de las regiones en las que están ubicadas. Por tal razón, ha aparecido la integración ambiental en los proyectos educativos institucionales de las universidades y en concreto a sus funciones de docencia, investigación, extensión y gestión (Mora Penagos, 2012). 
Bio - grafia. Escritos sobre la Biología y su Enseñanza. ISSN 2027

Edición Extraordinaria. p.p. 1275- 1288

Memorias del VIII Encuentro Nacional de Experiencias en Enseñanza de la Biología y la Educación Ambiental. III Congreso Nacional de Investigación en Enseñanza de la Biología.

\section{Metodología}

Para abordar el tema de la formación de docentes universitarios se propone el diseño pedagógico de una secuencia lógica y progresiva que según (Cañal, 1997), pueden ser llevadas a un nivel de transición en la inclusión de la dimensión ambiental, cómo se muestra en la tabla 1. Niveles de Transición para la formación de docentes e inclusión de la dimensión ambiental

Tabla 1 Niveles de Transición para la formación de docentes

$e$ inclusión de la dimensión ambiental

\begin{tabular}{|l|l|l|l|l|}
\hline $\begin{array}{c}\text { Niveles de } \\
\text { Transición }\end{array}$ & Capacidades & \multicolumn{1}{|c|}{ Temas } & Resultados & Secuencia \\
\hline Contextual & $\begin{array}{l}\text { Problematiza } \\
\text { r }\end{array}$ & $\begin{array}{l}\text { Relaciones del } \\
\text { desarrollo } \\
\text { socio- } \\
\text { económico en } \\
\text { contexto }\end{array}$ & $\begin{array}{l}\text { Diferencia de } \\
\text { saberes y } \\
\text { prácticas desde } \\
\text { los } \\
\text { componentes } \\
\text { principales de } \\
\text { la dimensión } \\
\text { ambiental }\end{array}$ & $\begin{array}{l}\text { Problemáticas } \\
\text { ambientales } \\
\text { reales desde la } \\
\text { crítica y el saber } \\
\text { ambiental }\end{array}$ \\
\hline Comprensivo & $\begin{array}{l}\text { Establece } \\
\text { relaciones } \\
\text { entre la } \\
\text { naturalezay } \\
\text { la sociedad }\end{array}$ & $\begin{array}{l}\text { Relaciones del } \\
\text { todo y sus } \\
\text { partes } \\
\text { subjetivas, con } \\
\text { el otro, lo }\end{array}$ & $\begin{array}{l}\text { Nueva visión de } \\
\text { ver el mundo. } \\
\text { No } \\
\text { fragmentada } \\
\text { con tendencia }\end{array}$ & $\begin{array}{l}\text { Análisis de } \\
\text { diferentes } \\
\text { tendencias } \\
\text { globales de lo lo } \\
\text { ambiental. Desde }\end{array}$ \\
\hline
\end{tabular}


Bio - grafia. Escritos sobre la Biología y su Enseñanza. ISSN 2027

Edición Extraordinaria. p.p. 1275- 1288

Memorias del VIII Encuentro Nacional de Experiencias en Enseñanza de la Biología y la Educación Ambiental. III Congreso Nacional de Investigación en Enseñanza de la Biología.

\begin{tabular}{|l|l|l|l|l|}
\hline & & $\begin{array}{l}\text { histórico, social } \\
\text { ycultural }\end{array}$ & humanizante & $\begin{array}{l}\text { la } \\
\text { responsabilidad, } \\
\text { protección. }\end{array}$ \\
\hline $\begin{array}{l}\text { De-construir } \\
\text { Y construir }\end{array}$ & $\begin{array}{l}\text { De-construir } \\
\text { y construir } \\
\text { conocimiento } \\
\text { sy prácticas }\end{array}$ & $\begin{array}{l}\text { Mitos, } \\
\text { artefactos, } \\
\text { concepciones, } \\
\text { paradigmas } \\
\text { simbolismos }\end{array}$ & $\begin{array}{l}\text { Secuencias } \\
\text { para diferentes } \\
\text { acciones de los } \\
\text { diversos grupos } \\
\text { sociales }\end{array}$ & $\begin{array}{l}\text { Diseño de } \\
\text { instrumentos } \\
\text { metodológicos } \\
\text { para la } \\
\text { enseñanza }\end{array}$ \\
\hline Referencial & $\begin{array}{l}\text { Construir } \\
\text { diferentes } \\
\text { conceptos y } \\
\text { teorías. }\end{array}$ & $\begin{array}{l}\text { Visiones lineal, } \\
\text { compleja, } \\
\text { transversal, } \\
\text { proyectiva, } \\
\text { Interdisciplinar } \\
\text { y } \\
\text { trasnsdisciplina } \\
\text { r }\end{array}$ & $\begin{array}{l}\text { Construir } \\
\text { discurso sobre } \\
\text { la práctica } \\
\text { educativa y la } \\
\text { relación con lo } \\
\text { ambiental } \\
\text { participativos }\end{array}$ & $\begin{array}{l}\text { La dialógica } \\
\text { entre los } \\
\text { enfoques } \\
\text { pedagógica }\end{array}$ \\
\hline
\end{tabular}

Fuente (Cardona, 2008)

Así mismo en la estructura de contenidos propios para un reconocimiento de saberes previos de los docentes, que incluye un ciclo de: describir, delimitar, establecer, estudiar, diseñar, elaborar, verificar y rediseñar los contenidos, como una secuencia didáctica de una unidad ambientalizada para que con el uso de las TIC, pueda ser la base de la formación permanente de los docentes universitarios. Ver figura 1. Secuencia didáctica de una unidad ambientalizada 
Bio - grafia. Escritos sobre la Biología y su Enseñanza. ISSN 2027

Edición Extraordinaria. p.p. 1275- 1288

Memorias del VIII Encuentro Nacional de Experiencias en Enseñanza de la Biología y la Educación Ambiental. III Congreso Nacional de Investigación en Enseñanza de la Biología.

Ya para el diseño pedagógico de los contenidos, (Cabero, 2006), presenta una base pedagógico en e-learning que permite definir los escenarios para la formulación de los objetivos del proceso de formación y construcción de un saber con el uso de la tecnología. Ver figura 2. Diseño Pedagógico de Contenidos para la formación de Docentes Universitarios Ambientalziados.

Figura 1. Secuencia Didáctica de una Unidad Ambientalizada. (Cañal, 1997) Proyectó: Cardona, 2014
$\begin{gathered}\text { Primera Aproximación a la Estructura de Contenidos } \\ \text { para la Formación de Docentes Universitarios } \\ \text { ambientalizados y mediados por las TIC }\end{gathered}$

Secuencia Didáctica de una Unidad Ambientalizada
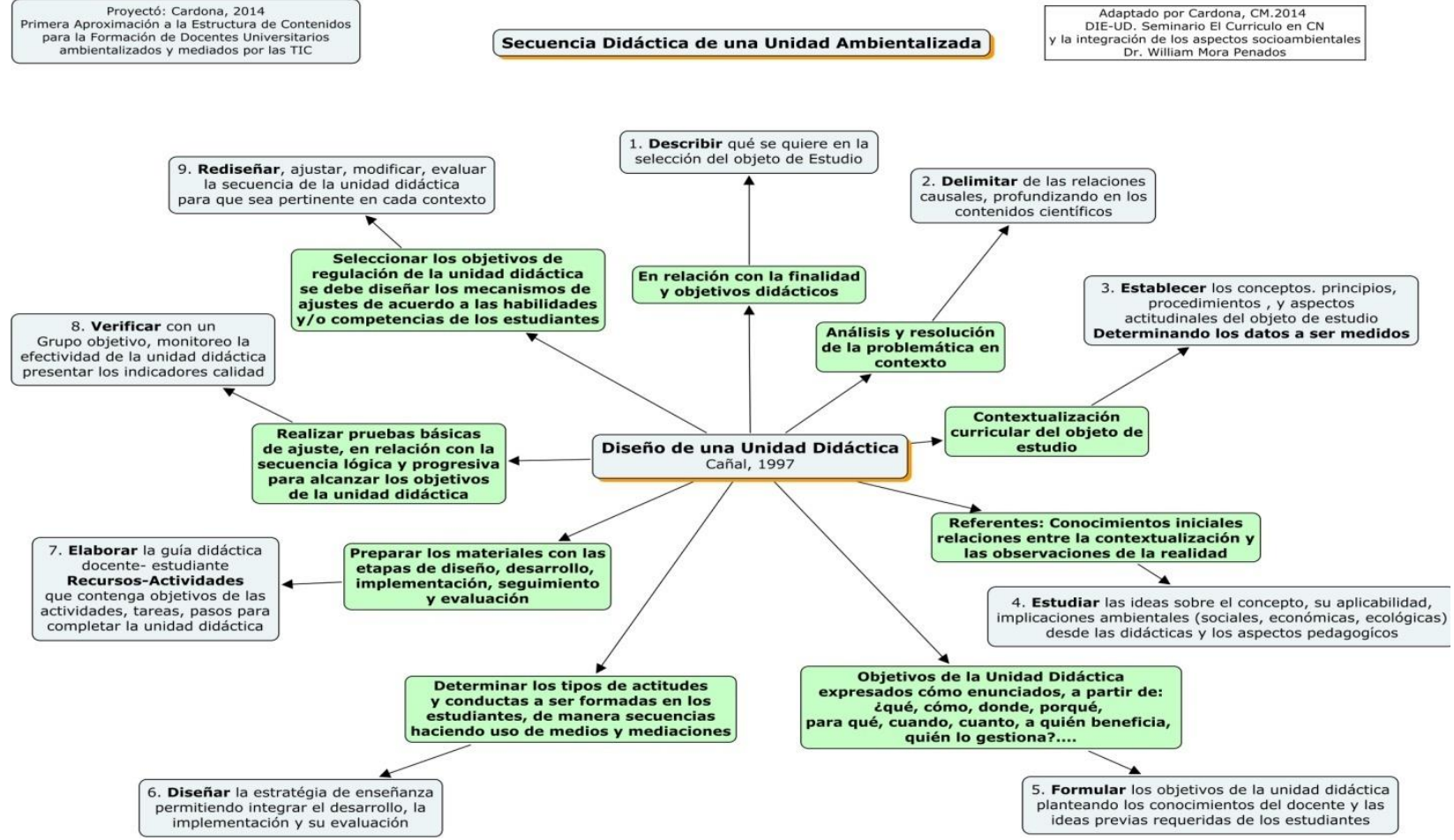
Bio - grafia. Escritos sobre la Biología y su Enseñanza. ISSN 2027

Edición Extraordinaria. p.p. 1275- 1288

Memorias del VIII Encuentro Nacional de Experiencias en Enseñanza de la Biología y la Educación Ambiental. III Congreso Nacional de Investigación en Enseñanza de la Biología.

Figura 2. Diseño pedagógico en la formación de docentes universitarios ambientalizados (Cardona, 2008)

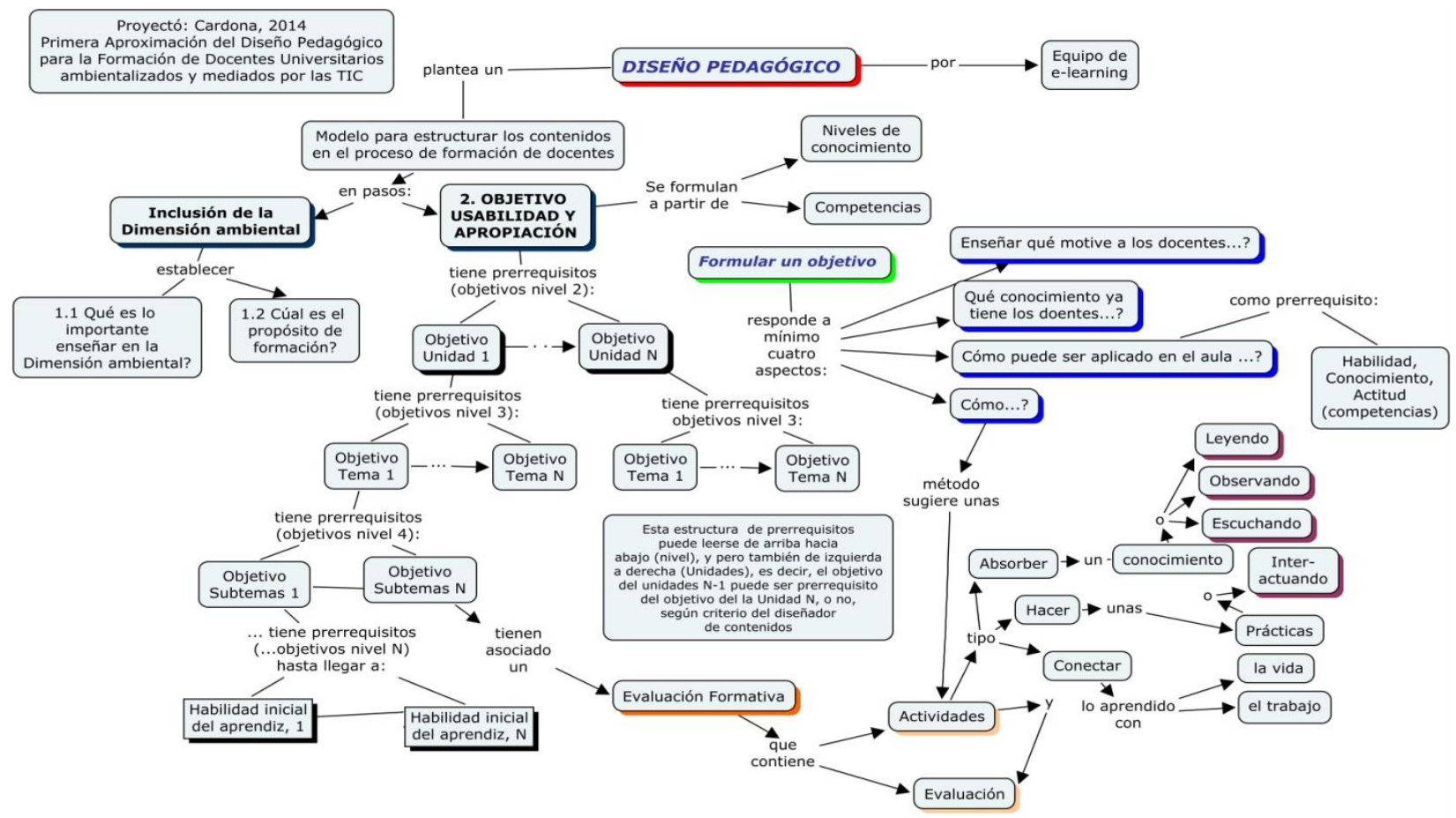


Bio - grafia. Escritos sobre la Biología y su Enseñanza. ISSN 2027

Edición Extraordinaria. p.p. 1275- 1288

Memorias del VIII Encuentro Nacional de Experiencias en Enseñanza de la Biología y la Educación Ambiental. III Congreso Nacional de Investigación en Enseñanza de la Biología.

\section{Resultados y Análisis}

Diseño de una unidad didáctica ambientaliazda y mediada por Tic en un contexto del "Agua como patrimonio hídrico para la planificación", se establecen los elementos de análisis y se publica en un ambiente virtual de aprendizaje, para colocarlo disponible en la formación permannete de docentes, se tocan todos los temas que involucra el conocimiento del agua, desde una estructura de contenidos por objetivos de aprendizaje, sólo se presneta la visión general del esquema diseñado y está en constrcción la evaluación del proceso de aprendizaje y la transerencia del saber a los curriculos particulares de los docentes en formación. Figura 3. Agua como patrimonio para la planifiacación.

Figura 3. Agua como patrimonio para la palnificación 
Edición Extraordinaria. p.p. 1275- 1288

Memorias del VIII Encuentro Nacional de Experiencias en Enseñanza de la Biología y la Educación Ambiental. III Congreso Nacional de Investigación en Enseñanza de la Biología.

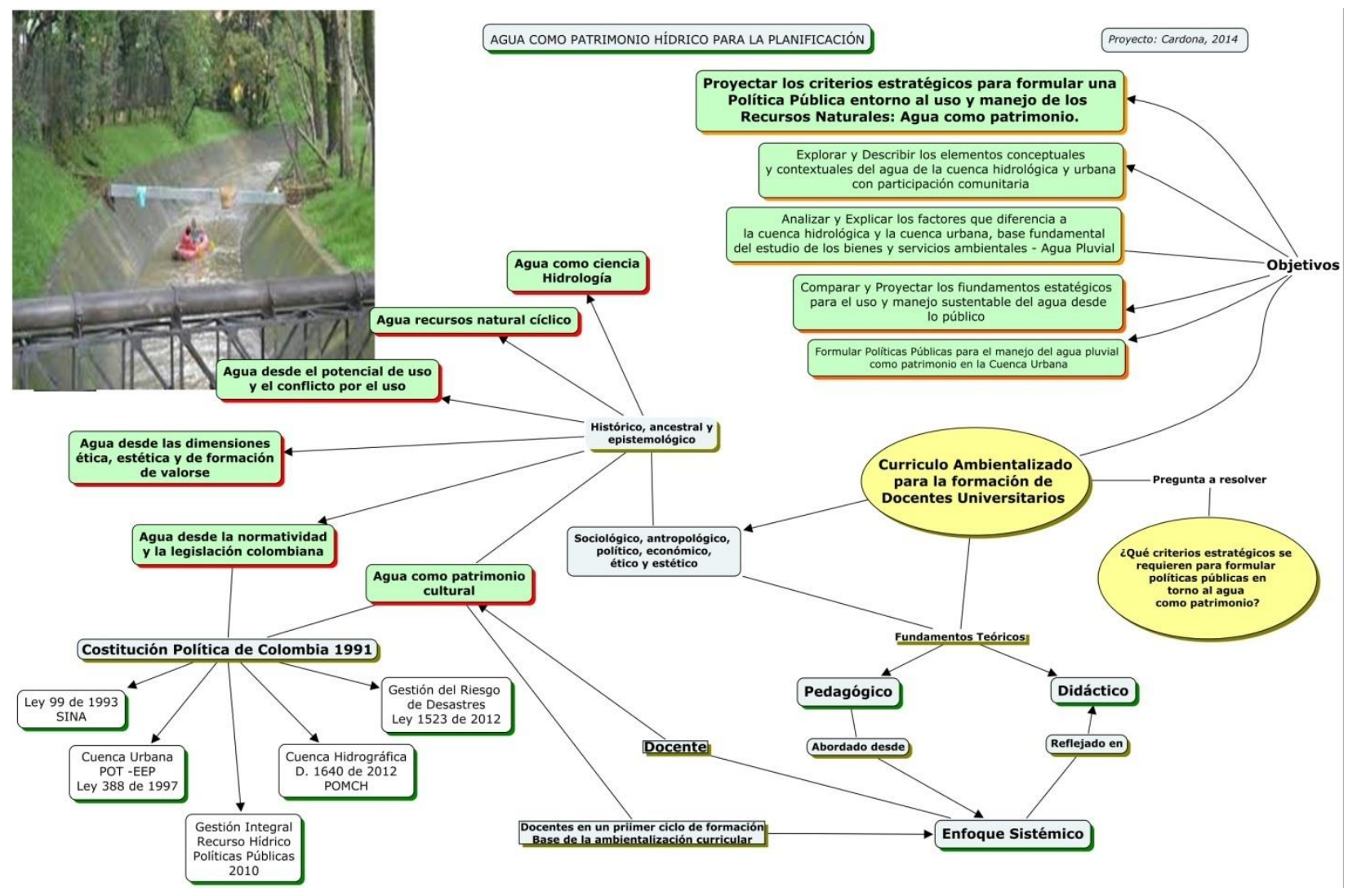

\section{Conclusiones}

Pretender establecer cómo construir un currículo ambientalizado, o cualquier componte que lo conforme, requiere de la valoración de diferentes posiciones y teorías sobre las concepciones pedagógicas y didácticas que pueden aportar en orientar el abordaje de las 
Bio - grafia. Escritos sobre la Biología y su Enseñanza. ISSN 2027

Edición Extraordinaria. p.p. 1275- 1288

Memorias del VIII Encuentro Nacional de Experiencias en Enseñanza de la Biología y la Educación Ambiental. III Congreso Nacional de Investigación en Enseñanza de la Biología.

situaciones problémicas ambientales desde el ámbito de las instituciones educativas y de aquellas que pretender promover una educación ambiental, esto implicará:

1. Un diálogo cultural, que incluye involucrar la herencia "occidental" de la ciencia, la tecnología vistas en lo académico desde un currículo disciplinar y las culturas locales. Este diálogo debe ser asumido como un proceso mediador que permita integrar las disciplinas hacia dimensiones que las integren y les permitan visualizar su pertinencia en relación acorde con lo natural, con los sujetos y colectivos en los que se encuentran inmersas las instituciones educativas.

2. Las fuentes para la construcción y cambios curriculares en las instituciones son los estudiantes, profesores y todo un sistema cultural en el cual se han construido como sujetos $y$ en el cual deben resignificarse para mejorar sus relaciones entre sí con lo natural.

3. El sistema educativo es un conjunto de elementos (subsistemas) que se relaciona con otros sistemas del entorno y establecen relaciones de intercambio de información, materia y energía constituyen diversas dinámicas de las cuales dependen.

4. Los procesos formación permanente de los docentes debe considerarse en la elaboración de prácticas educativas ambientalizadas abordan tanto procesos cognitivos y sociales propuestos en las teorías constructivistas así como de la pedagogía crítica que involucra el aprendizaje en sentido bidireccional del docente y del estudiante, que los reconoce como 
Bio - grafia. Escritos sobre la Biología y su Enseñanza. ISSN 2027

Edición Extraordinaria. p.p. 1275- 1288

Memorias del VIII Encuentro Nacional de Experiencias en Enseñanza de la Biología y la Educación Ambiental. III Congreso Nacional de Investigación en Enseñanza de la Biología.

sujetos culturales y visualiza la intencionalidad educativa en la preservación de la diversidad biológica y cultural.

5. Un práctica pedagógica ambientalizada requiere de involucrar a través de estrategias la participación de actores sociales: estudiantes, docentes y comunidad, esto permitirá realizar una interpretación real (hermenéutica) de las situaciones problemitas que los afectan, tomar posiciones y asumir decisiones en busca de avanzar hacia una solución.

6. La construcción de actividades hacia una práctica pedagógica ambientalizada, en este texto, parte de establecer una Situación problèmica objeto de estudio (¿para qué?), para ser contextualizada curricularmente, determinando los: conocimientos (¿qué?), objetivos (¿para qué?), actitudes, procedimientos así como los materiales requeridos para su desarrollo (¿Cómo? ¿Cuándo? ¿Con qué?) : Además luego de obtener un prime diseño se deben realizar pruebas de ajuste que se requieran y culminar en un rediseño pertinente y transferible (evaluación).

\section{Bibliografía}

Cabero Almenara, J. (2006). Bases pedagógicas del e-learning. Obtenido de $\underline{h t+p: / / r u s c . u o c . e d u / i n d e x . p h p / r u s c / a r t i c l e / v i e w F i l e / v 3 n 1-c a b e r o / v 3 n 1-c a b e r o ~}$

Cañal, .P. (1997). El diseño de una unidad didáctica: fundamentos y procedimientos. (D. Editores, Ed.) Investigación y enseñanza (7), 109-132. 
Bio - grafia. Escritos sobre la Biología y su Enseñanza. ISSN 2027

Edición Extraordinaria. p.p. 1275- 1288

Memorias del VIII Encuentro Nacional de Experiencias en Enseñanza de la Biología y la Educación Ambiental. III Congreso Nacional de Investigación en Enseñanza de la Biología.

Cardona, C. (2008). Verificación del cumplimiento de los objetivos educativos y de competencias en el aprendizaje: Estudio de caso; Asignatura cuencas Hidrográficas. Trabajo de grado (Sin publicar) , 1- 147.

Mora Penagos, W. M. (2012). La inclusión de la dimensión ambiental en la educación superior: Un estudio de caso en la facultad de medio ambiente de la Universidad Distrital de Bogotá.

Schutz, A. (1994). Le chercheur et le quotidien. Paris: Klincksieck. 